\title{
Imaging the Dynamic Release and Capture of Vesicle Membrane Proteins in Mammalian Cells
}

\author{
Justin W. Taraska, Kem A. Sochacki and Ben T. Larson
}

National Heart Lung and Blood Institute, National Institutes of Health, Bethesda, MD USA

Cells of the nervous system communicate with one another by releasing peptides, neurotransmitters, and small molecules by exocytosis. Triggered exocytosis in neurons and endocrine cells is a highly regulated process. Excitable cells go to great lengths to ensure that exocytosis occurs at precisely the right time and location and that the correct quantity of cargo is released during each exocytic burst. Once vesicle cargo such as lipids and proteins exits a vesicle and enters the plasma membrane it must be corralled and recaptured from the surface of the cell. A pathway proposed to be important for this process is clathrin-mediated endocytosis. Classically, clathrin, adapter proteins, and mechano-enzymes, have been suggested to assemble around vesicle material. This drives the formation of clathrin-coated pits which are able to internalize material into the cell. While many of the individual proteins important for this process have been identified, how these components capture vesicle material and assemble together to drive the formation of an endocytic vesicle is not well understood.

To study the pathway of protein release and recapture from exocytic vesicles we imaged the postfusion dynamics of a single vesicle membrane protein, the vesicular acetylcholine transporter (VaChT), from individual exocytic vesicles in living neuroendocrine cells with total internal reflection fluorescence microscopy. We combine these measurements with super-resolution interferometric photoactivation localization microscopy (iPALM), transmission electron microscopy, and modeling to map the nanometer-scale topography and architecture of the plasma membrane structures responsible for the transporter's recapture [1]. We show that after exocytosis, VaChT rapidly diffuses into the plasma membrane but travels only a short distance before it is corralled over a dense network of membraneresident clathrin structures. We propose that the extreme density of these structures acts as a short-range diffusion trap. They quickly sequester diffusing materials and limit its spread across the membrane. This system could provide a means for clathrin-mediated endocytosis to quickly recycle exocytic proteins in highly excitable cells.

Next, because the complete molecular identity of these resident clathrin-coated structures are unknown, we developed a novel high-throughput two-color total internal reflection fluorescence microscopy and automated image processing pipeline to map a library of 78 proteins proposed to have roles in membrane trafficking at these sites [2]. With this method we determine both the local and cellwide distributions of these proteins at the plasma membrane. These results provide a unified systemslevel spatial map of the protein landscape of individual endocytic sites and a new framework for the unbiased spatial analysis of cellular systems. With these data we identify a core group of proteins that associate with clathrin-coated structures (CCS) in PC12 cells. We also observe a broad range in the degree of associations. Furthermore, most proteins were found to be distributed amongst these structures and across the membrane in a well-mixed random pattern with no apparent underlying spatial organization.

Our combined imaging results illustrate the ability of fluorescence microscopy and quantitative analysis to interrogate the underlying structure of a complex spatial system and screen for new 
associations and patterns within the cell. The random distributions we observe for the proteins studied suggests an overall model for how the plasma membrane is organized in relation to endocytic structures in neuroendocrine cells. CCSs are very dense, from 2 to 11 times as dense as reported in other cell types. This dense network of CCSs may allow for robust rapid sorting and capture of cargo and a possible enhancement in signal transduction. The network map we generate can also be used as a reference for comparison between different cell types, states, and perturbations. Investigating individual functional units of interacting molecules in their cellular context will deepen our understanding of the selforganization, complexity, spatial organization, and control of biological systems.

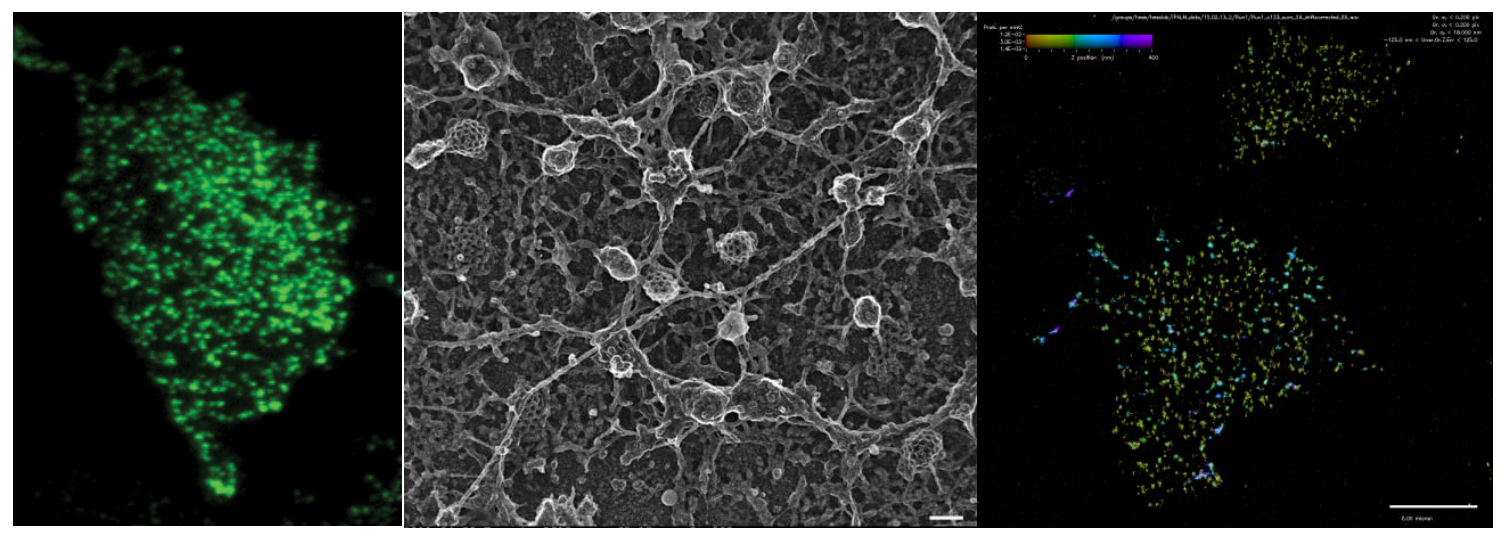

Figure 1. High resolution imaging of endocytic sites in neuroendocrine PC12 cells. On the left is a TIRF image of a living PC12 cell expressing a GFP-tagged protein that marks single endocytic sites. In the center is a transmission electron micrograph of a platinum replica of the inner membrane of a PC12 cell (Scale bar is $100 \mathrm{~nm}$ ). Individual clathrin coated pits can be seen to stud the membrane. On the right is a super-resolution 3D iPALM image of labeled clathrin in the same cells (Scale bar is 5 microns).

\section{References:}

[1] Sochacki KA, Larson BT, Sengupta DC, Daniels MP, Shtengel G, Hess HF, Taraska JW, Nature Communications. 3 (2012), p. 1154.

[2] Larson BT, Sochacki KA, Kindem JM, Taraska JW, Mol. Biol. Cell. 25(2014), p.2084-93

[3] The authors acknowledge funding from the Intramural Research Program of the National Heart Lung and Blood Institute, National Institutes of Health, USA. 